AIAA-2005-5229

17th Computational Fluid Dynamics Conference, June 6-9, Toronto, Canada

\title{
A Study of Multigrid Preconditioners Using Eigensystem Analysis
}

\author{
Thomas W. Roberts* and R. C. Swanson ${ }^{\dagger}$ \\ Mail Stop 128, NASA Langley Research Center, Hampton, VA 23681-2199
}

\begin{abstract}
The convergence properties of numerical schemes for partial differential equations are studied by examining the eigensystem of the discrete operator. This method of analysis is very general, and allows the effects of boundary conditions and grid nonuniformities to be examined directly. Algorithms for the Laplace equation and a two equation model hyperbolic system are examined.
\end{abstract}

\section{Introduction}

One of the critical needs in computational fluid dynamics is the development of rapidly converging algorithms. Present Reynolds averaged Navier-Stokes (RANS) schemes have exceedingly poor convergence rates on realistic problems. Understanding the source of slow convergence should lead to improvements in the algorithms. Although many sources of poor convergence are understood in a general, qualitative way (e.g., grid stretching and skewness), the exact nature of these difficulties in practical problems is often a mystery.

The most common tool for analyzing the stability and convergence of numerical methods is Fourier analysis, especially von Neumann stability analysis. ${ }^{1}$ This is applicable to uniform, Cartesian grids and constant coefficient equations, and neglects the effects of boundary conditions. Despite these seemingly severe restrictions, Fourier analysis is remarkably useful and leads to predictions of behavior that frequently are good quantitatively as well as qualitatively, even for real, nonlinear problems. However, boundary conditions can have a profound effect on the performance of a numerical scheme, and such effects cannot be determined with Fourier analysis. Understanding more general difficulties, such as the effects of stretched and unstructured grids, grid cell aspect ratio, and variable coefficient equations, requires more general analysis tools.

One approach is to look at the eigensystem of a flow solver. Eriksson and Rizzi ${ }^{2}$ used this approach to examine the behavior of their multistage, central difference Euler solver. Linearizing about a partially converged state, they used Arnoldi's method to extract the slowest converging eigenmodes. In this way they were able to examine in detail the persistent modes of the scheme. At the time of their work, computer speeds and memory sizes were such that it was extremely expensive to extract a handful of modes. Present day hardware, as well as improved algorithms for extracting the eigenmodes of large systems, ${ }^{3}$ makes their approach more viable. Furthermore, if a Krylov method such as GMRES is used as the iterative method, finding the eigenvalues and eigenvectors is very little additional work and provides a good diagnostic capability. ${ }^{4}$ On the other hand, this approach can be too general: it is not amenable to studying numerical difficulties in isolation and to evaluating the effectiveness of different components of an algorithm. One would like to be able to study simpler model problems, which may be clumsy to handle with a full-blown flow solver.

Current computer speed and memory, and the availability of software packages such as MATLAB ${ }^{\circledR}$, makes it possible to compute the complete eigensystems of moderately sized discretizations of partial differential equations. If the iterative method is to be used as a preconditioner for a Krylov method, knowledge of the

\footnotetext{
${ }^{*}$ Research Scientist, Computational AeroSciences Branch (Presently at Aurora Flight Sciences, Inc.)

$\dagger$ Research Scientist, Computational AeroSciences Branch. Senior Member AIAA.

Copyright (C) 2005 by the American Institute of Aeronautics and Astronautics, Inc. The U.S. Government has a royalty-free license to exercise all rights under the copyright claimed herein for Governmental purposes. All other rights are reserved by the copyright owner.
} 
spectrum can allow one to make good estimates for the convergence of the Krylov iteration. ${ }^{5}$ In the present work, we explicitly calculate the eigensystems of general model equations. We restrict ourselves to linear systems, but allow for nonuniform grids and arbitrary boundary conditions.

In the next section, a general description of iterative methods for solving linear systems is presented. Although this material is not new, it provides a convenient framework for developing the analysis tools. Multigrid preconditioning is described in some detail as a prelude to our examples. In this paper we illustrate the use of the eigensystem analysis by examining the Laplace equation and a two equation model hyperbolic system. The results for the Laplace equation show significant differences with the usual Fourier analysis. Analysis is presented for an implicit scheme and a multistage scheme applied to the two equation system.

\section{Structure of iterative schemes}

All linear, two level iterative methods for the solution of the system $L q=f$ can be viewed as preconditioned iterative methods. This includes classical relaxation methods such as Jacobi and Gauss-Seidel, as well as explicit and implicit time marching methods. In this section, the connection between most of the common methods for solving systems of linear equations arising from discretizations of partial differential equations is made explicit. Only linear systems of equations are considered. Although we are interested in discrete approximations to partial differential equations, the discussion is quite general. What follows is not new, but the authors have not seen this idea presented in this fashion previously. Lomax and Steger ${ }^{6}$ discussed iterative methods in computational fluid dynamics in a very general way, which comes close to what follows herein. However, they did not make explicit the connection between the power series approximation to the inverse of a linear operator and most of the common methods for solving the system of equations.

\section{A. Power series approximation to the inverse}

Consider the function $y=1-x$, where $x$ is a real or complex scalar. When $|x|<1$, then the binomial theorem gives the power series representation of the inverse of $y$,

$$
\begin{aligned}
y^{-1}=\frac{1}{1-x} & =1+x+x^{2}+\cdots \\
& =\sum_{k=0}^{\infty} x^{k} .
\end{aligned}
$$

This notion can be extended to a linear operator in an $m$ dimensional space. First, consider the $m \times m$ matrix $A$. If $\operatorname{det}(I-A) \neq 0$, where $I$ is the identity matrix, and $\|A\|<1$, then

$$
\begin{aligned}
(I-A)^{-1} & =I+A+A^{2}+\cdots \\
& =\sum_{k=0}^{\infty} A^{k}
\end{aligned}
$$

This power series representation of the inverse of a linear operator is especially attractive when $m$ is large and $A$ is sparse. A direct inversion of $I-A$ may not be feasible either in storage or time. Although $A$ is sparse, the inverse $(I-A)^{-1}$ generally is not. If the series in Eq. (1) converges rapidly, then the inverse can be computed in $O(m)$ operations.

Consider the system $L q=f$. To invert this system iteratively, one almost invariably is required to precondition the system. Let $P$ be the preconditioner, which is an $m \times m$, nonsingular matrix. The preconditioned system to be solved is $P L q=P f$. By choosing the preconditioner such that $\|I-P L\|<1$, then $P L=I-(I-P L)$ has the form in Eq. (1) with $A=I-P L$. This leads to the power series representation of $L^{-1}$

$$
L^{-1}=\sum_{k=0}^{\infty}(I-P L)^{k} P .
$$

The preconditioner $P$ may be thought of as an approximate inverse to $L$. The closer $P$ is to $L^{-1}$, the more rapid will be the convergence of the iteration. The goal is to find a $P$ that is not only a good approximation to $L^{-1}$, but is sparse and easy to evaluate. In practice the preconditioner $P$ is not usually formed explicitly. 
Let $L_{n}^{-1}=\sum_{k=0}^{n}(I-P L)^{k} P$, the $n$-term approximation to the inverse of $L$. Successive approximations to $L^{-1}$ satisfy the recurrence equation

$$
L_{n}^{-1}=P+(I-P L) L_{n-1}^{-1}
$$

In practice, we are interested in the solution to the system $L q=f$ rather than $L^{-1}$ itself. It is usual to compute the corresponding sequence of approximations to $q$,

$$
\begin{aligned}
q_{n} & =L_{n}^{-1} f \\
& =P f+(I-P L) L_{n-1}^{-1} f \\
& =P f+(I-P L) q_{n-1} .
\end{aligned}
$$

It is seen that the solution $q$ is a fixed point of the iteration (4), and that the sequence converges to the solution of the original problem.

If a sequence of $\nu$ preconditioners $P_{k}$ is used to construct $L_{n}^{-1}$,

$$
L_{n-\nu+k}^{-1}=P_{k}+\left(I-P_{k} L\right) L_{n-\nu+k-1}^{-1}, \quad k=1 \ldots \nu,
$$

then we can introduce a preconditioner $\tilde{P}_{\nu}$ defined by the recurrence equation

$$
\tilde{P}_{1}=P_{1}, \quad \tilde{P}_{k}=P_{k}+\left(I-P_{k} L\right) \tilde{P}_{k-1}, \quad k=2 \ldots \nu
$$

which allows us to write

$$
L_{n}^{-1}=\tilde{P}_{\nu}+\left(I-\tilde{P}_{\nu} L\right) L_{n-\nu}^{-1}
$$

The sequence of $\nu$ preconditioning stages can be interpreted as subiterations, or an inner cycle, and $\tilde{P}_{\nu}$ is the preconditioner of the outer iteration or cycle. This recursive structure can be used to build a complex preconditioner from a general sequence of simpler ones. For example, a multigrid preconditioner is built up from a sequence of fine grid smoothing and coarse grid correction preconditioning steps.

Although we have treated only left preconditioning, the above discussion applies as well to right preconditioning, i.e., $L P P^{-1} q=f$, where $L P$ rather than $P L$ is the preconditioned operator.

\section{B. Illustrative examples of common iterative methods}

Common relaxation schemes are constructed by splitting the operator $L$ into a sum of the form $L=M-N$, where $M$ is nonsingular. Then an iterative scheme is

$$
M q_{n}=f+N q_{n-1} .
$$

Familiar examples are to take $M$ to be the diagonal elements of $L$, which yields Jacobi iteration, or to take $M$ to be the lower triangular part of $L$, which yields lexicographic Gauss-Seidel. Solving this equation gives

$$
\begin{aligned}
q_{n} & =M^{-1} f+M^{-1} N q_{n-1} \\
& =M^{-1} f+M^{-1}(M-L) q_{n-1} \\
& =M^{-1} f+\left(I-M^{-1} L\right) q_{n-1} .
\end{aligned}
$$

Comparing Eq. (6) to Eq. (4), it is immediately apparent that this is a preconditioned iterative scheme where $M^{-1}$ is the preconditioner.

If $L$ is the steady state part of a time dependent operator, $\partial_{t} q+L q=f$, then the solution to $L q=f$ can be obtained by marching in time until the asymptotic state is reached. This is the most common solution method in computational fluid dynamics. If explicit, forward time integration is used, and $q_{n}$ is the solution at time $t=n \tau$, then the iteration is

$$
q_{n}=\tau f+(I-\tau L) q_{n-1} .
$$

This has the form of Eq. (4) with a scalar preconditioner $\tau$. If local time steps are used to accelerate convergence, then $\tau$ is a diagonal matrix with the local time steps along the diagonal. Similarly, explicit 
multistage schemes and two level implicit schemes can be put in the form of Eq. (4). For example, the three stage explicit scheme

$$
\begin{aligned}
q^{(0)} & =q_{n-1}, \\
q^{(1)} & =q^{(0)}+\alpha_{1} \tau\left(f-L q^{(0)}\right), \\
q^{(2)} & =q^{(0)}+\alpha_{2} \tau\left(f-L q^{(1)}\right), \\
q^{(3)} & =q^{(0)}+\tau\left(f-L q^{(2)}\right), \\
q_{n} & =q^{(3)}
\end{aligned}
$$

is equivalent to the preconditioned scheme (4) with $P=\tau I-\alpha_{2} \tau^{2} L+\alpha_{1} \alpha_{2} \tau^{3} L^{2}$. Finally, consider the implicit approximate factorization scheme

$$
\left(I+\tau L_{1}\right)\left(I+\tau L_{2}\right)\left(q_{n}-q_{n-1}\right)=\tau\left(f-L q_{n-1}\right),
$$

where $L=L_{1}+L_{2}$. The preconditioner of this system is $P=\left(I+\tau L_{2}\right)^{-1}\left(I+\tau L_{1}\right)^{-1} \tau$.

\section{Multigrid preconditioning}

The preconditioners in subsection II. B. are straightforward to write down explicitly. In more general cases the recursive expression Eq. (5) may be used to build an expression for the preconditioner corresponding to any arbitrary scheme in a straightforward way. We use this to construct the preconditioner corresponding to a general multigrid operator.

A multigrid iteration consists of two parts, fine grid smoothing and a coarse grid correction. It is typical to parameterize a multigrid cycle by three numbers: $\nu_{1}$, the number of smoothing iterations before the coarse grid correction; $\nu_{2}$, the number of smoothing iterations after the coarse grid correction; and the cycle index $\gamma$, which is the number of coarse grid iterations in the cycle. A cycle index of one corresponds to a $V$-cycle, two corresponds to a $W$-cycle.

We wish to construct the preconditioner $P_{m}$ of a general multigrid cycle. The pre- and post-smoothing iterations have the same recursive structure shown in Eq. (4). Let $P_{s}$ be the preconditioner corresponding to the the particular smoother being used. We start to build $P_{m}$ by the iteration

$$
\begin{gathered}
P_{m}=0 \\
\text { for } k=1 \text { to } \nu_{1} \text { do } \quad P_{m} \leftarrow P_{s}+\left(I-P_{s} L\right) P_{m} .
\end{gathered}
$$

Next a coarse grid correction is determined. Let $P_{c}$ be the preconditioner corresponding to a coarse grid update, i.e., the approximate inverse to the coarse grid operator $L_{c}$. Let $\mathcal{R}$ be the restriction operator (transfers data from a fine grid to a coarse grid) and $\mathcal{P}$ be the prolongation operator (transfers data from a coarse grid to a fine grid). The contribution to the preconditioner, $P_{\gamma}$, for a cycle index $\gamma$ is

$$
\tilde{P}_{c}=0
$$

$$
\begin{gathered}
\text { for } k=1 \text { to } \gamma \text { do } \quad \tilde{P}_{c} \leftarrow P_{c}+\left(I-P_{c} L_{c}\right) \tilde{P}_{c} . \\
P_{\gamma}=\mathcal{P} \tilde{P}_{c} \mathcal{R} .
\end{gathered}
$$

This is used to update $P_{m}$ :

$$
P_{m} \leftarrow P_{\gamma}+\left(I-P_{\gamma} L\right) P_{m} .
$$

After the coarse grid correction is computed, $\nu_{2}$ smoothing sweeps finish the cycle:

$$
\text { for } k=1 \text { to } \nu_{2} \text { do } \quad P_{m} \leftarrow P_{s}+\left(I-P_{s} L\right) P_{m} \text {. }
$$

One multigrid cycle may be written $q_{n}=P_{m} f+\left(I-P_{m} L\right) q_{n-1}$.

The preconditioner $P_{m}$ can be written explicitly by expanding the terms in Eqs.(8) to (11), which yields

$$
\begin{aligned}
P_{m}=\sum_{k=0}^{\nu_{2}-1}(I- & \left.P_{s} L\right)^{k} P_{s}+\left(I-P_{s} L\right)^{\nu_{2}} \\
& \times\left(\sum_{k=0}^{\nu_{1}-1}\left(I-P_{s} L\right)^{k} P_{s}+\mathcal{P}\left(\sum_{k=0}^{\gamma-1}\left(I-P_{c} L_{c}\right)^{k} P_{c}\right) \mathcal{R}\left(I-L \sum_{k=0}^{\nu_{1}-1}\left(I-P_{s} L\right)^{k} P_{s}\right)\right) .
\end{aligned}
$$


Because of the recursive nature of the multigrid cycle, the computation of the coarse grid preconditioner $P_{c}$ is done in the same way as $P_{m}$.

The convergence of the multigrid scheme can be analyzed by determining the spectrum of the amplification matrix $G_{m}=I-P_{m} L$. The asymptotic convergence rate is the modulus of the largest eigenvalue of $G_{m}$.

\section{Model equation analysis}

The algorithm for the computation of $P_{m}$ and $G_{m}$ has been programmed in MATLAB ${ }^{\circledR}$ for two model equations: the Laplace equation and a two equation hyperbolic system. The discrete operator $L$ and the preconditioners $P$ are represented explicitly as matrices. The spectrum of $G_{m}$ is computed using the built-in function eig.

\section{A. Two dimensional Laplace equation}

The first example we consider is the Laplace equation in two space dimensions,

$$
\partial_{x}^{2} q+\partial_{y}^{2} q=0
$$

on the unit square with Dirichlet boundary conditions. Equation (13) is discretized with the standard five point central difference stencil. The difference equation is solved using multigrid with lexicographic point Gauss-Seidel as the smoother. As a point of reference, we initially take a uniform discretization in the $x$ and $y$ directions, so that a comparison with Fourier analysis may be made.

The convergence of this scheme is usually estimated by smoothing analysis. ${ }^{7}$ Letting $\theta_{x}$ and $\theta_{y}$ be the Fourier modes in the $x$ and $y$ directions, respectively, the amplification factor $g$ of one sweep of the Gauss-Seidel smoother is

$$
g\left(\theta_{x}, \theta_{y}\right)=1-\frac{2\left(\cos \theta_{x}-1\right)+2\left(\cos \theta_{y}-1\right)}{e^{-i \theta_{x}}+e^{-i \theta_{y}}-4} .
$$

The modulus of the amplification factor $|g| \leq 1 / 2$ in the range $\pi / 2 \leq \theta_{x} \leq 3 \pi / 2, \pi / 2 \leq \theta_{y} \leq 3 \pi / 2$. This is the smoothing rate. Because the coarse grid is effective on the long wavelength error, the multigrid asymptotic convergence rate is estimated as the smoothing rate to the power $\nu_{1}+\nu_{2}$, the total number of fine grid relaxation sweeps per cycle.

A comparison of the spectra for lexicographic Gauss-Seidel relaxation and multigrid with the result of Fourier analysis is shown in Figure 1. Equation (13) is discretized on a uniform, $33 \times 33$ grid. The spectrum of the matrix operator for Gauss-Seidel with periodic boundary conditions is seen to compare extremely well with the result of Fourier analysis, as it should. When Dirichlet boundary conditions are introduced, however, the spectrum of the operator changes quite dramatically. The eigenvalues are more tightly clustered about the origin, and the slowly converging modes have collapsed to the positive real axis. This is qualitatively much different than the picture given by the Fourier analysis. When Gauss-Seidel is used as a smoother for a $V(1,0)$ multigrid cycle $\left(V(1,0)\right.$ means $\left.\nu_{1}=1, \nu_{2}=0\right)$ on five grids, all the eigenvalues are now well inside the limit given by the smoothing analysis. Another effect of the coarse grid correction is that the eigenvalues are not quite so tightly clustered near the origin.

To confirm the validity of the analysis, Table 1 shows the convergence rates predicted from consideration of the maximum eigenvalue, and the actual convergence rates of the iterative solver for homogeneous Dirichlet boundary conditions and random initial conditions. The rates agree extremely well. We have also considered a four stage multistage scheme as the smoother, treating the Laplace operator as the steady state asymptote of the heat equation. A comparison of the convergence rates for this scheme with the predicted values is shown in Table 2. The standard multistage coefficients were chosen, and the time step was at the stability limit given by von Neumann analysis. Again, the agreement between the actual and predicted convergence rates is excellent.

One of the advantages of this analysis is that we can examine the effect of variable coefficients, such as arise from grid stretching. Equation (13) is discretized on a unit square with uniform spacing in the $x$-direction and a stretching rate of 1.2, a severe stretching, in the $y$-direction. The aspect ratio of the grid cells, $h_{y} / h_{x}$, varies from 0.0188 at the lower boundary to 5.349 at the upper boundary. This is a case that cannot be treated by Fourier analysis. The effect of grid stretching is shown in Figure 2, where both the Gauss-Seidel smoother alone and a $V(1,0)$ cycle are compared to the uniform grid case. The degradation of the convergence rate is apparent, but about $90 \%$ of the eigenvalues have a modulus less than $1 / 2$. Except for 
Table 1. Predicted and actual multigrid convergence rates for the solution of the Laplace equation on a uniform $33 \times 33$ grid using lexicographic Gauss-Seidel smoothing and homogeneous Dirichlet boundary conditions

\begin{tabular}{cclcc}
\hline \hline & \multicolumn{2}{c}{$V(1,0)$ cycle } & \multicolumn{2}{c}{$W(1,0)$ cycle } \\
Grids & Actual & Predicted & Actual & Predicted \\
\hline 1 & 0.9901 & 0.9904 & - & - \\
2 & 0.9530 & 0.9530 & 0.9170 & 0.9170 \\
3 & 0.8191 & 0.8191 & 0.5006 & 0.5006 \\
4 & 0.4658 & 0.4658 & 0.3033 & 0.3016 \\
5 & 0.3314 & 0.3318 & 0.2975 & 0.3016 \\
\hline \hline
\end{tabular}

Table 2. Predicted and actual multigrid convergence rates for the solution of the Laplace equation on a uniform $33 \times 33$ grid using four stage multistage smoothing and homogeneous Dirichlet boundary conditions

\begin{tabular}{cclcc}
\hline \hline & \multicolumn{2}{c}{$V(1,0)$ cycle } & \multicolumn{2}{c}{$W(1,0)$ cycle } \\
Grids & Actual & Predicted & Actual & Predicted \\
\hline 1 & 0.9952 & 0.9952 & - & - \\
2 & 0.9764 & 0.9764 & 0.9579 & 0.9579 \\
3 & 0.9074 & 0.9074 & 0.7153 & 0.7153 \\
4 & 0.7116 & 0.7116 & 0.6038 & 0.6053 \\
5 & 0.6028 & 0.6058 & 0.6033 & 0.6053 \\
\hline \hline
\end{tabular}

the persistent modes lying along the positive real axis, the spectra are qualitatively similar to their uniform grid counterparts.

\section{B. Two equation hyperbolic system}

The second example considered is the model two equation system

$$
\begin{array}{r}
\partial_{t} u-\kappa \partial_{x} u-\partial_{y} v=0, \\
\partial_{t} v+\partial_{x} v-\partial_{y} u=0 .
\end{array}
$$

This is a particularly simple hyperbolic model system. When the parameter $\kappa=1$, steady solutions to the system (15) satisfy the Cauchy-Riemann equations. If $\kappa$ is taken to be the Prandtl-Glauert factor, steady solutions to Eq. (15) can be interpreted as the velocity components of a small perturbation compressible flow. ${ }^{\mathrm{a}}$

To solve this system, we initially consider the implicit scheme described by Roberts and Warren ${ }^{8,9}$ to discretize the system. First order upwind differencing is used on the left hand side, and second order upwind biased differencing is used on the right hand side. Using Fourier analysis, Roberts and Warren showed that if a direct inversion of the left hand side operator is performed, all wavelengths (except for $\theta_{x}=0, \theta_{y}=0$ ) have an amplification factor of less than $1 / 2$. In practice, the left hand side is not directly inverted, but must be solved approximately. Red-black Gauss-Seidel iteration is used to approximately invert the first order left hand side operator. This is taken as the smoother for a multigrid cycle for the full system. The number of Gauss-Seidel subiterations of the first order system is an additional parameter of the iterative scheme.

Figure 3 shows a comparison of spectra for Eq. (15) with $\kappa=1$ on a uniform $32 \times 32$ grid. Ten redblack subiterations were used to invert the first order operator and the CFL number was $10^{6}$. The upper left plot shows the eigenvalues for a direct inversion of the left hand side. The dashed circle shows the maximum amplification factor given by Roberts and Warren. ${ }^{8}$ On the upper right, the spectrum for ten red-black iterations and no multigrid is shown. The shape is very close to that for a direct inversion, with the exception of a few outliers. Incorporating this smoother into a five level $V(1,0)$ multigrid cycle gives

\footnotetext{
${ }^{a}$ However, Eq. (15) is not the correct system of unsteady small perturbation compressible flow.
} 
Table 3. Predicted and actual multigrid convergence rates for the solution of two equation hyperbolic system on a uniform $32 \times 32$ grid using ten red-black relaxation sweeps to invert the left hand side

\begin{tabular}{ccccccc}
\hline \hline & \multicolumn{2}{c}{$V(1,0)$ cycle } & \multicolumn{2}{c}{$W(1,0)$ cycle } & \multicolumn{2}{c}{$W(1,1)$ cycle } \\
Grids & Actual & Predicted & Actual & Predicted & Actual & Predicted \\
\hline 1 & 0.7210 & 0.7201 & - & - & - & - \\
2 & 0.6361 & 0.6361 & 0.6568 & 0.6570 & 0.2603 & 0.2598 \\
3 & 0.6683 & 0.6689 & 0.6660 & 0.6658 & 0.2595 & 0.2598 \\
4 & 0.7373 & 0.7373 & 0.6676 & 0.6671 & 0.2606 & 0.2598 \\
5 & 0.7295 & 0.7295 & 0.6657 & 0.6663 & 0.262 & 0.2598 \\
\hline \hline
\end{tabular}

the plot on the lower left. Although there is more clustering of the eigenvalues near the origin, a large number of them lie outside the dashed circle, leading to no improvement in the asymptotic convergence rate. Finally, a direct solve of the coarse grid problem gives the two grid spectrum on the lower right, which differs qualitatively from the $V$-cycle spectrum. The two grid convergence rate is 0.5649 , which is still not as good as single grid results with a direct inversion of the left hand side.

A comparison of the convergence rates for the system using various numbers of grids and cycles is shown in Table 3. All results are for a uniform $32 \times 32$ grid, $\kappa=1$, a CFL number of $10^{6}$ and ten red-black subiterations of the first order operator as the smoother. The observed convergence rates for an iterative solution of Eq. (15) with homogeneous characteristic boundary conditions and random initial conditions are shown for comparison. The comparison of the predicted and actual rates are in excellent agreement. Interestingly, the addition of more grid levels does not improve the convergence rate. In fact, for the $V(1,0)$ cycle the convergence deteriorates when more than one coarse grid level is used. The $W(1,1)$ cycle is completely insensitive to additional coarse grid levels.

We now consider a scheme recently introduced by Rossow. ${ }^{10}$ This scheme uses an explicit Runge-Kutta method as a framework. An implicit stage is included in each Runge-Kutta stage in order to extend stability and appropriately treat geometrically stiff discrete systems, which generally occur in numerically resolving flows with boundary layers. The implicit operator corresponds to a first-order approximation of the governing flow equations, and the full operator is approximately inverted iteratively with Gauss-Seidel relaxation. In the present work, unless otherwise indicated, an upwind biased discretization of the explicit residual function was implemented as described by Roberts and Warren. For the analysis we used a 5-stage Runge-Kutta scheme and set the CFL number to 100. The coefficients for the Runge-Kutta scheme were taken from Van Leer et al., ${ }^{11}$ and they are as follows: 0.0695, 0.1602, 0.2898, 0.5060, 1 . Lexicographic (Lex), Red-Black (R-B), and symmetric (Sym) Gauss-Seidel (G-S) iterations were considered for the implicit operator inversion.

In the the upper plots of Figure 4 spectra for the Runge-Kutta scheme applied to the hyperbolic system of Eq. (15) on the uniform $32 \times 32$ grid are shown. For comparison purposes consider also the corresponding spectra for the implicit scheme in Figure 3. Both schemes use red-black Gauss-Seidel iterations for approximately inverting the implicit operator. Here the implicit stage of the Runge-Kutta scheme uses three iterations. With the Runge-Kutta scheme as the smoother for the $V(1,0)$ multigrid cycle, there is significantly stronger eigenvalue clustering, as evident in the upper right plot. This improved clustering is reflected in the convergence rates presented in Table 4. As exhibited with the implicit scheme, additional grid levels in the multigrid provide little or no improvement in the asymptotic convergence rate.

The effect of mesh refinement on the uniform grid is also included in Figure 4. For the lower plots the analysis was applied on a $64 \times 48$ grid. The spectral patterns for the two grids are quite similar, differing primarily in density. There is the expected slowdown in single grid convergence rate (from 0.8002 to 0.8965 ). With the $V(1,0)$ multigrid cycle the convergence rate is 0.4508 on both meshes.

Figure 5 shows the effect of grid aspect ratio alone on the spectra of the Runge-Kutta scheme with $V(1,0)$ multigrid. In addition, the impact of different types of Gauss-Seidel relaxation is indicated. The number inside the parentheses denotes the number of iterations performed in each implicit stage. The aspect ratio is 100 throughout a $32 \times 32$ grid. With six iterations for approximate inversion of the implicit operator, the asymptotic convergence rates with lexicographic and red-black Gauss-Seidel are 0.6237 and 0.6450 , respectively. In the lower right plot we see that there is a strong effect on the spectrum clustering 
Table 4. Predicted and actual multigrid convergence rates for the solution of two equation hyperbolic system on a uniform $32 \times 32$ grid using a five stage R-K scheme, with an implicit stage corresponding to each explicit stage. Three red-black relaxation sweeps are used to invert the implicit operator.

\begin{tabular}{ccccccc}
\hline \hline & \multicolumn{2}{c}{$V(1,0)$ cycle } & \multicolumn{2}{c}{$W(1,0)$ cycle } & \multicolumn{2}{c}{$W(1,1)$ cycle } \\
Grids & Actual & Predicted & Actual & Predicted & Actual & Predicted \\
\hline 1 & 0.8000 & 0.8002 & - & - & - & - \\
2 & 0.5237 & 0.5240 & 0.4522 & 0.4522 & 0.2039 & 0.2038 \\
3 & 0.4507 & 0.4508 & 0.4524 & 0.4524 & 0.2038 & 0.2039 \\
4 & 0.4504 & 0.4508 & 0.4524 & 0.4524 & 0.2040 & 0.2039 \\
5 & 0.4508 & 0.4508 & 0.4524 & 0.4524 & 0.2039 & 0.2039 \\
\hline \hline
\end{tabular}

with symmetric Gauss-Seidel, resulting in a convergence rate of 0.4579 . Although not shown, even an aspect ratio of 1000 resulted in the same rate of convergence. This suggests that there is at most a weak sensitivity to aspect ratio.

In general a good preconditioner needs to be effective not only on a grid with aspect ratio but also a grid with stretching. While Fourier analysis is not appropriate for stretched grids, the matrix analysis discussed herein can easily handle general stretchings in all coordinate directions. We now consider the effect of grid stretching on the performance of the Runge-Kutta scheme. For this purpose a $32 \times 32$ affine grid with variable spacing in the $\mathrm{x}$ - and $\mathrm{y}$-directions was generated. The grid was defined on a rectangular domain with $-5 \leq x \leq 5$ and $0 \leq y \leq 5$. Points in the $\mathrm{x}$-direction were distributed with a tangent stretching function on the intervals $-5 \leq x \leq-1 / 2,1 / 2 \leq x \leq-5$, and a cosine spacing on the interval $-1 / 2 \leq x \leq 1 / 2$. In the $\mathrm{y}$-direction the grid was stretched with a rate of 1.2 on the interval $0 \leq y \leq 5$. The grid aspect ratio, $h_{x} / h_{y}$, varies from about 700 to about $1 / 90$ over the computational domain.

The spectra for the Runge-Kutta scheme applied to the stretched grid are displayed in Figure 6. The implicit operator was either approximately inverted with red-black Gauss-Seidel or symmetric Gauss-Seidel. For both types of Gauss-Seidel the same computational work was done. There is stronger eigenvalue clustering and fewer outliers with the symmetric iteration. Nevertheless, with the $V(1,0)$ multigrid cycle there is good clustering for both red-black and symmetric iteration. This suggests that with multigrid both versions of the Runge-Kutta scheme would be amenable to a Krylov method (e.g., GMRES). As smoothers for the multigrid, the two versions of the Runge-Kutta scheme have nearly the same maximum eigenvalue in modulus (0.7259 for red-black iteration and 0.7594 for symmetric iteration). To verify these predicted convergence rates, calculations were performed on the same grid used in the analysis for inviscid flow over a parabolic bump. A perturbation boundary condition was imposed at the solid boundary and the initial solution was set to zero. The convergence rates for the computations with the two variations of the Runge-Kutta scheme were essentially the same as the predicted rates.

So far we have examined the performance of the Runge-Kutta scheme with only the wide discretization stencil (21 points in two dimensions) of reference. ${ }^{8}$ In the application of the Runge-Kutta scheme Rossow used a narrow second order upwind differencing stencil (9 points). A question arises concerning the effect of the difference stencil type, especially since Roberts and Warren showed that a first-order implicit scheme with a narrow pure upwind second order stencil for the right-hand side has poor damping properties. In Figure 7 the spectra of the Runge-Kutta scheme for the aspect ratio(AR) of 100 grid and the stretched grid are displayed. Symmetric Gauss-Seidel was used to invert the implicit operator in all cases. The upper plots in the figure are for the $\mathrm{AR}=100$ grid, and the lower plots are for the stretched grid. In comparing the upper right plot with the lower right plot of Figure 5, one can see that the eigenvalue patterns for the two stencils are different. Nevertheless, for each stencil all the eigenvalues lie within the radius $=1 / 2$ circle. There is somewhat faster $V(1,0)$ multigrid convergence with the narrow stencil (rate $=0.3876$ ) than with the wide stencil (rate $=0.4579$ ). For the stretched grid the differences between the eigenvalue patterns for the narrow and wide stencils can be seen by comparing the lower plots of Figures 6 and 7 . The convergence rates corresponding to the narrow stencil are again somewhat faster. With the single grid the narrow stencil rate is 0.8538 , and the wide stencil rate is 0.8735 . For the $V(1,0)$ multigrid the convergence rates with the narrow and wide stencils are 0.6900 and 0.7594 , respectively. 


\section{Summary}

It has been shown that two level iterative schemes for the solution of a linear system $L q=f$ can be interpreted as solution to the preconditioned system $P L q=P f$ by a power series expansion for $(P L)^{-1}$. This includes not only classical relaxation methods, but also two level time marching schemes for the unsteady equation $\partial_{t} q+L q=f$. Using this common structure leads to a simple algorithm for the computation of the preconditioner and amplification matrix. The spectrum of the latter can be efficiently found for moderately sized problems. This provides a very general and flexible way to study the convergence properties of numerical methods for partial differential equations.

The two model equations presented here are illustrative of the use of the method. The authors are particularly interested in using the eigensystem analysis to address the convergence difficulties of the NavierStokes equations on highly stretched grids. The initial steps in extending the analysis to the compressible Navier-Stokes equations have been taken. Once this extension is completed we plan to consider the analysis for unstructured grids, to which Fourier methods do not apply.

\section{Acknowledgment}

The authors would like to thank Dr. Cord Rossow for helpful discussions concerning his Runge-Kutta scheme.

\section{References}

\footnotetext{
${ }^{1}$ Richtmyer, R. D., Morton, K. W., Difference Methods for Initial-Value Problems. New York: Interscience, 1967.

${ }^{2}$ Eriksson, L. E., Rizzi, A., "Computer-Aided Analysis of the Convergence to Steady State of Discrete Approximations to the Euler Equations," J. Comput. Phys., vol. 57, pp. 90-128, 1985.

${ }^{3}$ Sorensen, D. C., "Implicit Application of Polynomial Filters in a K-Step Arnoldi Method," SIAM J. Matrix Anal. Appl., vol. 13, pp. 357-85, 1992.

${ }^{4}$ Campobasso, M. S., Giles, M. B., "Effects of Flow Instabilities on the Linear Analysis of Turbomachinery Aeroelasticity," J. Propulsion and Power, vol. 19, no. 2, pp. 250-259, March-April 2003.

${ }^{5}$ Driscoll, T. A., Toh, K.-C., Trefethen, L. N., "From Potential Theory to Matrix Iterations in Six Steps," SIAM Rev., vol. 40, pp. 547-78, 1998.

${ }^{6}$ Lomax, H., Steger, J. L, "Relaxation Methods in Fluid Mechanics," Annu. Rev. Fluid Mech., vol. 7, pp. 63-88, 1975.

${ }^{7}$ Brandt, A., "Multigrid Techniques: 1984 Guide with Applications to Fluid Dynamics," GMD-Studie 85, GMD-FIT, 1985.

${ }^{8}$ Roberts, T. W., Warren, G. P., "Analysis of Implicit Second-Order Upwind-Biased Stencils," AIAA Paper 93-3379, June 1993.

${ }^{9}$ Warren, G. P., Roberts, T. W., "Multigrid Properties of Upwind-Biased Data Reconstruction," Sixth Copper Mountain Conference on Multigrid Methods, NASA Conference Publication 3224, Part 2, pp. 663-677, April 1993.

${ }^{10}$ Rossow, C.-C., "Convergence Acceleration for Solving the Compressible Navier-Stokes Equations," AIAA Paper 20050094, Jan. 1993.

${ }^{11}$ Van Leer, B., Tai, C.-H., Powell, K. G, "Design of Optimally Smoothing Multi-Stage Schemes for the Euler Equations," AIAA Paper 89-1933, June 1989.
} 


\section{Gauss-Seidel, Fourier analysis}

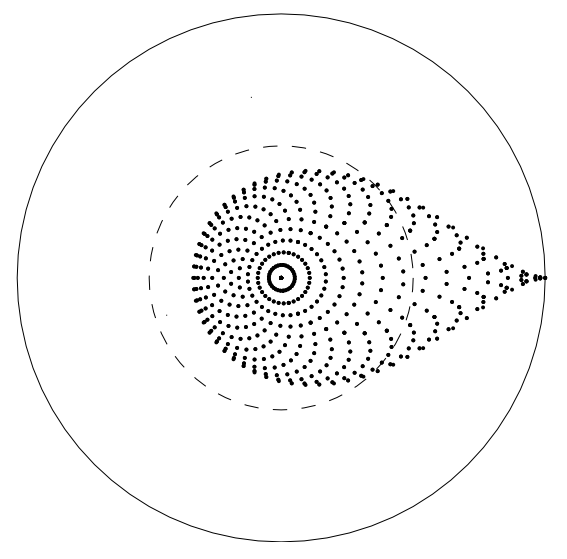

Gauss-Seidel, Dirichlet boundary

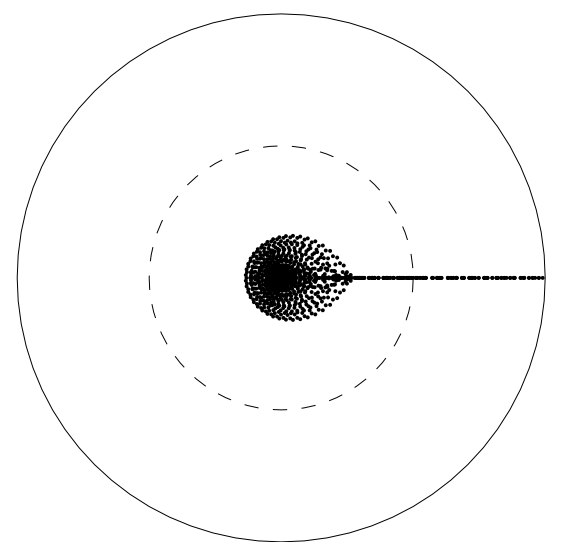

Gauss-Seidel, periodic boundary

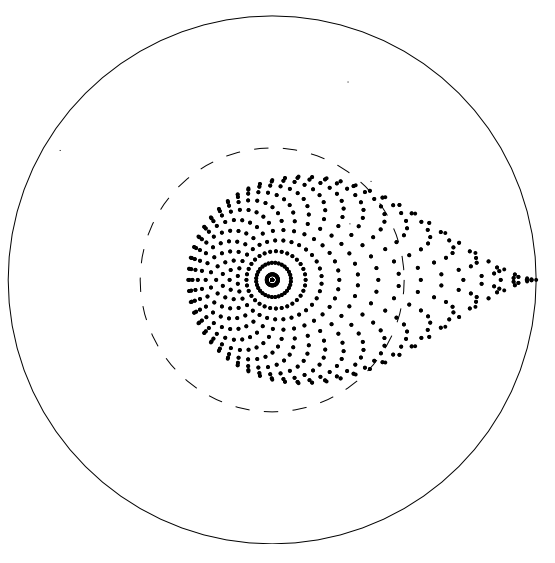

$\mathrm{V}(\mathbf{1}, \mathbf{0})$, Dirichlet boundary

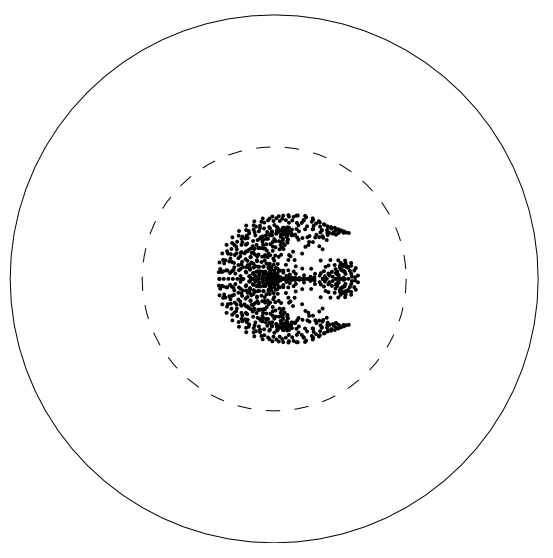

Figure 1. Spectra of preconditioners for the Laplace equation on a $33 \times 33$ grid. Solid line is the unit circle, dashed line is the smoothing rate given by Fourier analysis. 


\section{Gauss-Seidel, uniform grid}

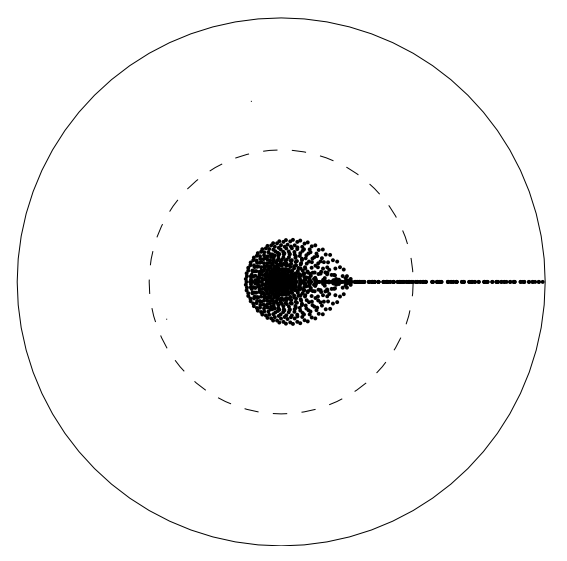

Gauss-Seidel, stretched grid

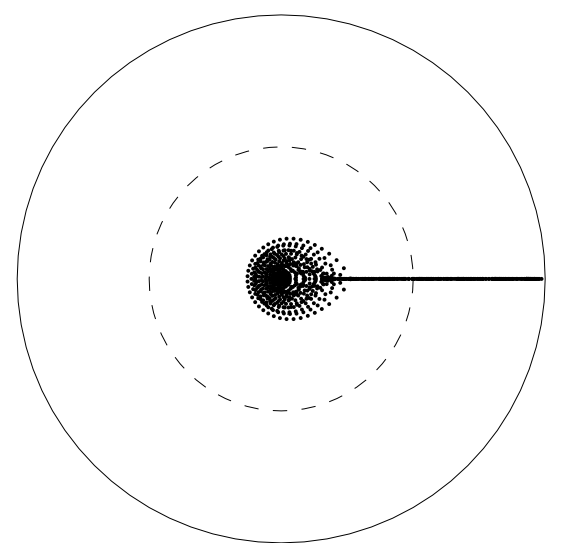

$\mathrm{V}(1,0)$, uniform grid

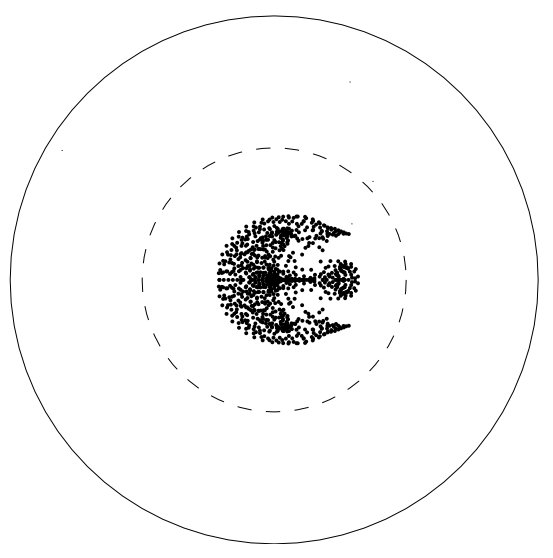

$\mathrm{V}(1,0)$, stretched grid

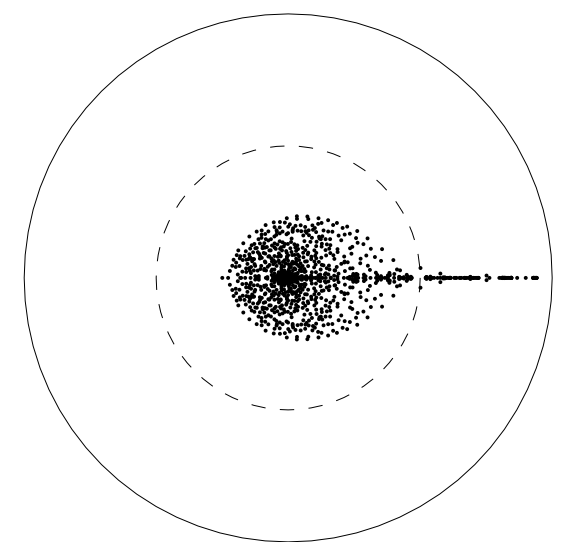

Figure 2. Spectra of preconditioners for the Laplace equation on a uniform and a stretched $33 \times 33$ grid. Solid line is the unit circle, dashed line is the smoothing rate given by Fourier analysis. 
Direct inversion of left-hand side

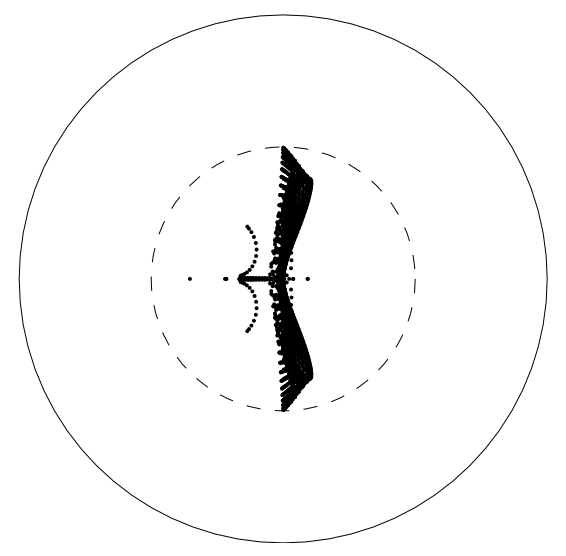

$V(1,0), 10$ subiterations

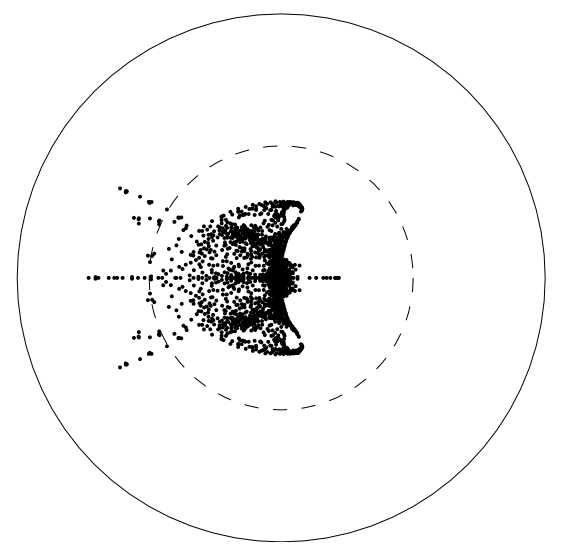

Red-Black Gauss-Seidel, 10 subiterations

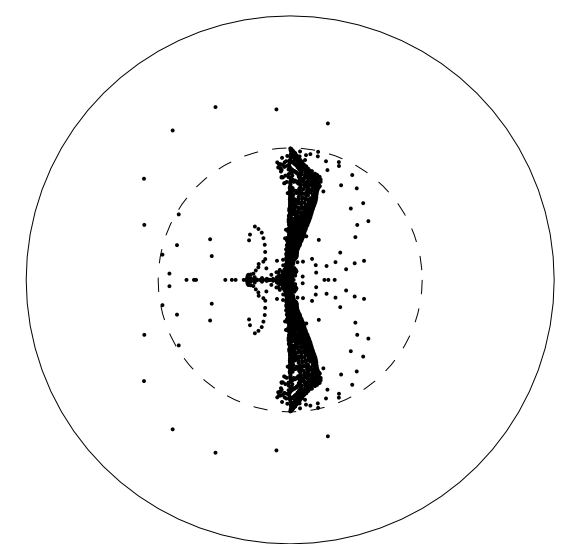

Two-grid $(1,0), 10$ subiterations

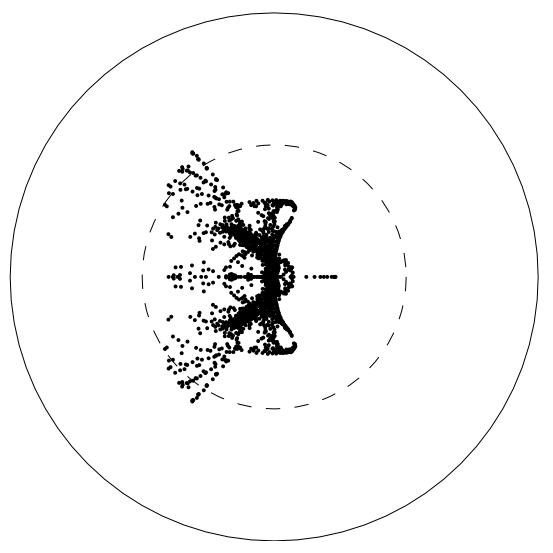

Figure 3. Spectra for the two equation system on a uniform $32 \times 32$ grid. Solid line is the unit circle, dashed line is amplification factor for a direct inversion of the first order left hand side operator. 


\section{Runge-Kutta (R-B G-S, 3)}

$32 \times 32$

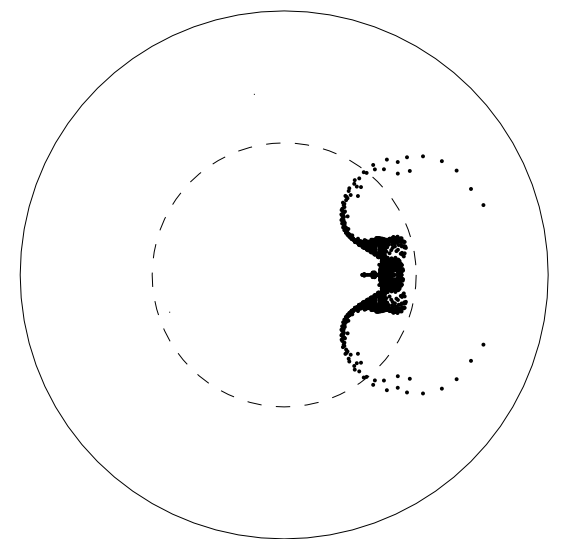

Runge-Kutta (R-B G-S, 3)

$64 \times 48$

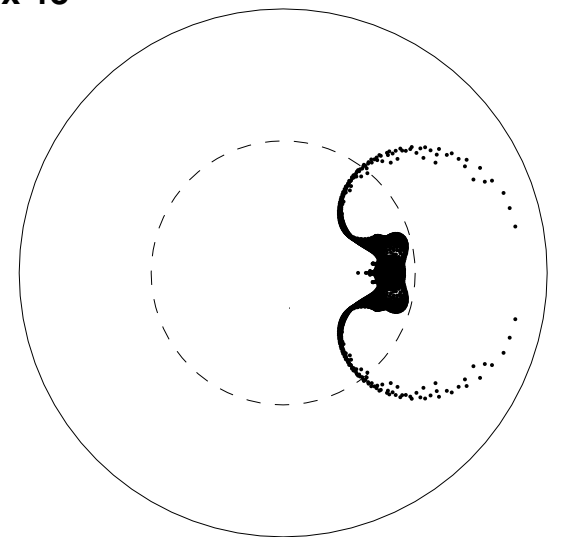

\section{Runge-Kutta (R-B G-S, 3), V(1,0)}

$32 \times 32$

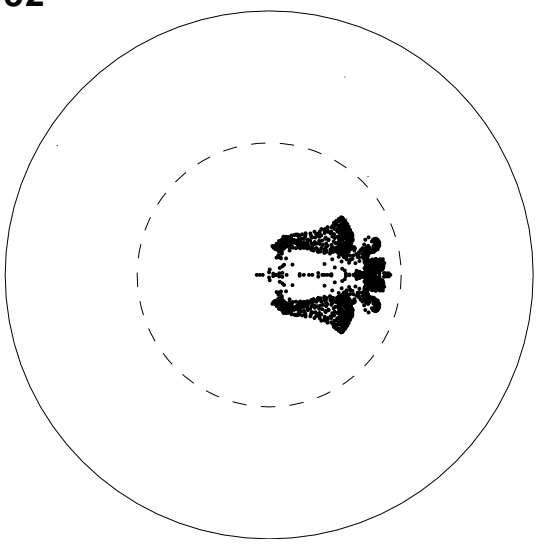

Runge-Kutta (R-B G-S, 3), V(1,0)

$64 \times 48$

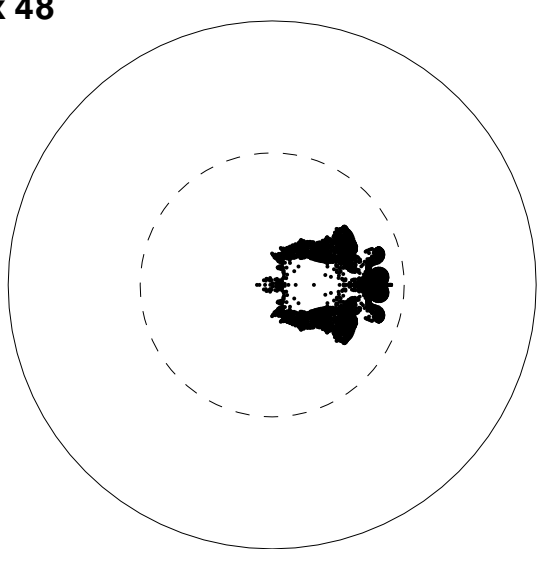

Figure 4. Spectra for the two equation system on uniform $32 \times 32$ and $64 \times 48$ grids. Solid line is the unit circle, dashed line corresponds to amplification factor of 0.5 . 
Runge-Kutta (R-B G-S, 3), V(1,0)

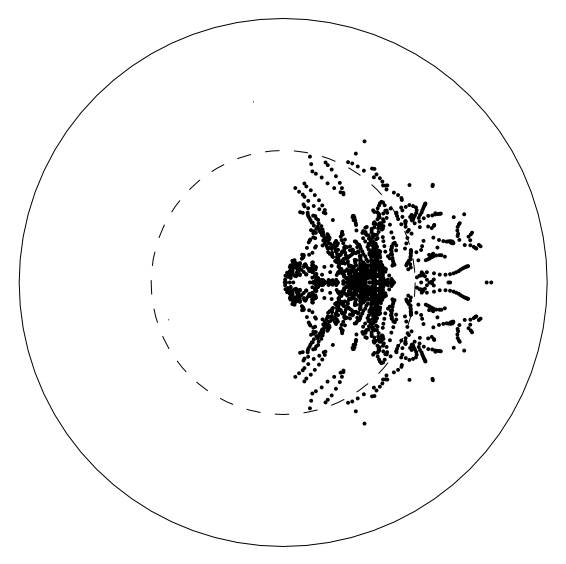

Runge-Kutta (Lex G-S, 6), V(1,0)

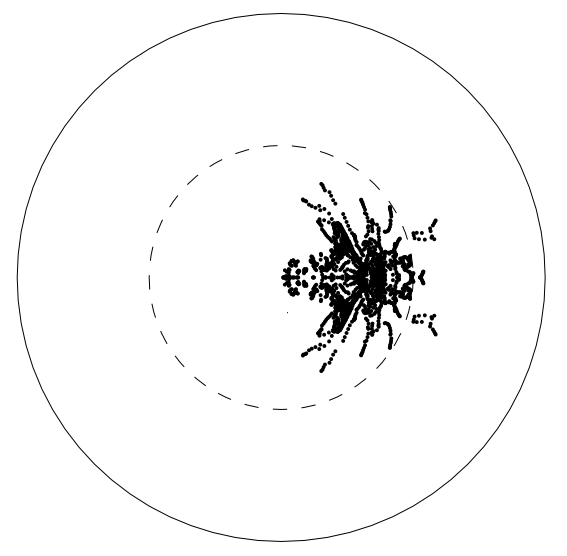

Runge-Kutta (R-B G-S, 6), V(1,0)

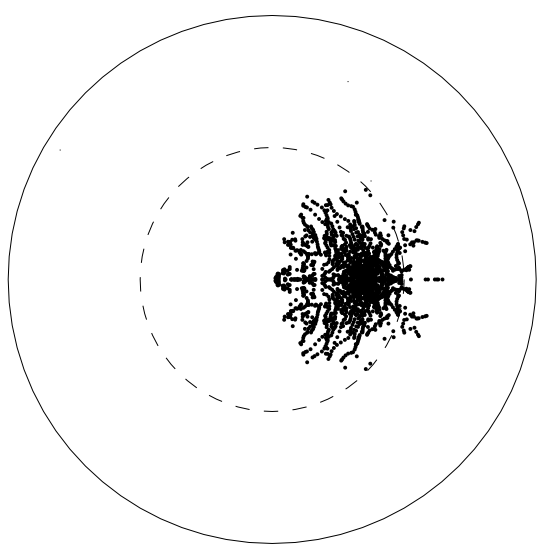

Runge-Kutta (Sym G-S, 3), V(1,0)

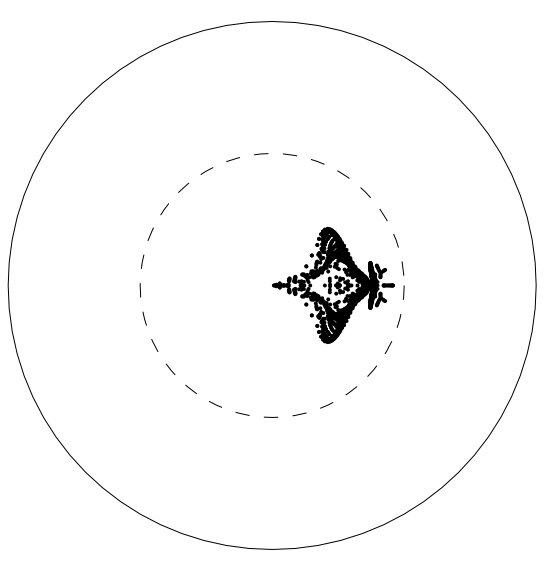

Figure 5. Spectra for the two equation system on a uniform, aspect ratio of $100,32 \times 32$ grid. All cases for $V(1,0)$ multigrid cycle. Solid line is the unit circle, dashed line corresponds to amplification factor of 0.5 . 
Runge-Kutta (R-B G-S, 6)

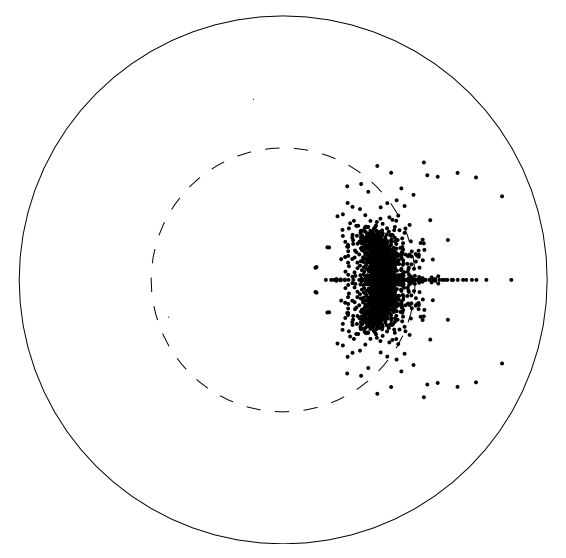

Runge-Kutta (Sym G-S, 3)

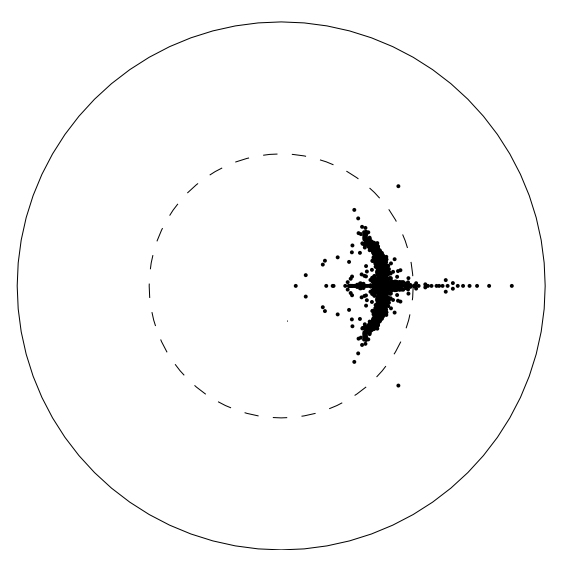

Runge-Kutta (R-B G-S, 6), V(1,0)

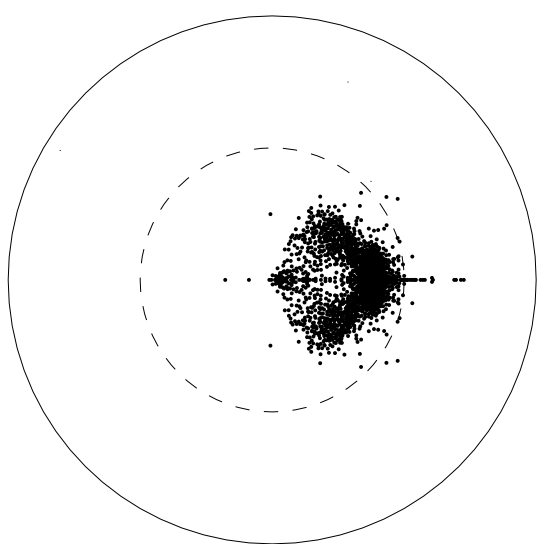

Runge-Kutta (Sym G-S, 3), V(1,0)

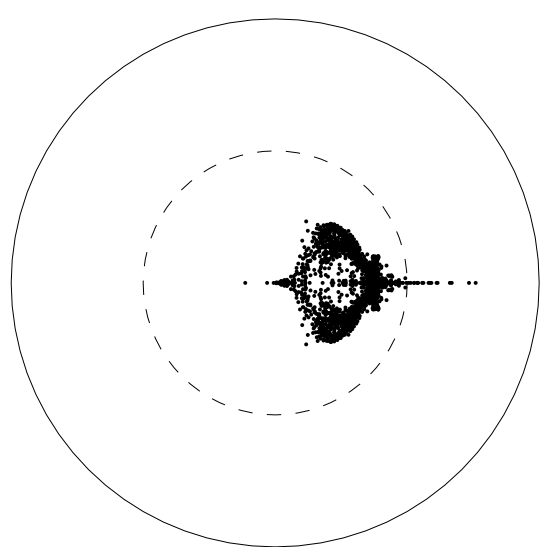

Figure 6. Spectra for the two equation system on a stretched $32 \times 32$ grid. Grid stretched in $x$ - and $y$-directions. Solid line is the unit circle, dashed line corresponds to amplification factor of 0.5 . 
Runge-Kutta (Sym G-S, 3)

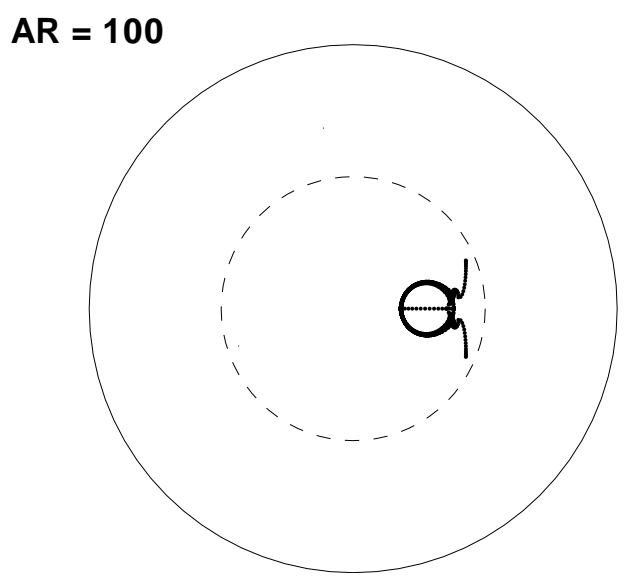

Runge-Kutta (Sym G-S, 3)

\section{Stretched}

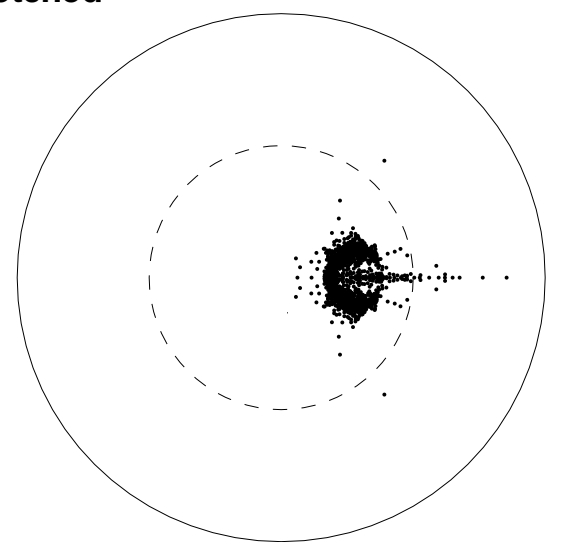

Runge-Kutta (Sym G-S, 3), V(1,0)

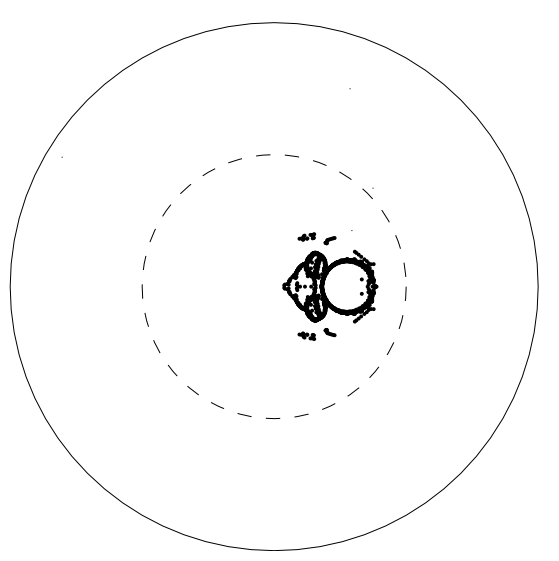

Runge-Kutta (Sym G-S, 3), V(1,0)

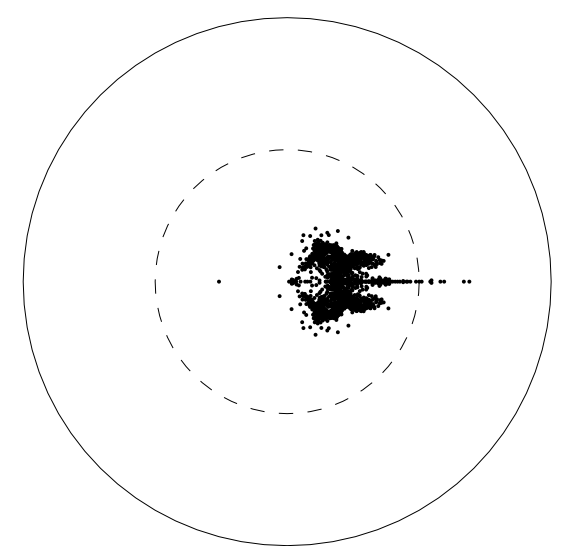

Figure 7. Spectra for the two equation system on a $32 \times 32$ grid. Standard nine point upwind differencing used for discretization. Top two plots for aspect ratio $A R=100$ grid; bottom two plots for stretched grid. Solid line is the unit circle, dashed line corresponds to amplification factor of 0.5 . 